\title{
Further Aspects of Weak Interaction Dynamics
}

\author{
Eliahu Comay \\ Charactell Ltd., Tel-Aviv, Israel \\ Email: elicomay@post.tau.ac.il
}

How to cite this paper: Comay, E. (2017) Further Aspects of Weak Interaction Dynamics. Open Access Library Journal, 4: e3397. https://doi.org/10.4236/oalib.1103397

Received: January 23, 2017

Accepted: February 21, 2017

Published: February 24, 2017

Copyright $\odot 2017$ by author and Open Access Library Inc.

This work is licensed under the Creative Commons Attribution International License (CC BY 4.0).

http://creativecommons.org/licenses/by/4.0/

\begin{abstract}
This work discusses properties of a recently published weak interactions theory which is derived from a Lagrangian density $\mathcal{L}_{w}$. This theory depends on the experimentally confirmed massive neutrino. The interaction which is carried by an appropriate mediating field is consistent with the Fermi coupling constant of weak interactions $G_{F}$. Its results prove the existence of a vector term $\boldsymbol{V}$ and an axial vector term $\boldsymbol{A}$ in a description of weak interactions processes and of their associated parity nonconservation. An analysis of the weak interactions Lagrangian density $\mathcal{L}_{w}$ shows similarity and differences between the theoretical structure of electrodynamics and that of weak interactions.
\end{abstract}

\section{Subject Areas}

Theoretical Physics

\section{Keywords}

Dynamics of Weak Interactions, Lagrangian Density, Equations of Motion

\section{Introduction}

This work is dedicated to some fundamental properties of weak interactions. An important part of an examination of the validity of a given physical theory is the search for vital elements that are not included in it. For example, every theory of an electrically charged particle should have an expression for a divergenceless 4-current $j_{, \mu}^{\mu}=0$, which is known by the name of the continuity equation. Indeed, a divergenceless 4-current is the differential form of charge conservation (see [1], p. 76). Charge conservation has a solid experimental support and it is a crucial feature of Maxwellian electrodynamics. Since the continuity equation represents charge conservation, one concludes that a quantum theory is unacceptable if it has no consistent expression for the continuity equation of its electrically charged particles. It turns out that a vital element of the Standard 
Model electroweak theory is the existence of a pair of electrically charged elementary particles, called $W^{+}$and $W^{-}$, respectively. As a matter of fact, the electroweak theory has no consistent expression for a divergenceless 4-current of its $W^{+}, W^{-}$particles. For more details of this issue, see Section 2 of [2] and references therein. This is just one reason justifying the claim that the electroweak theory is unacceptable and that weak interactions need an alternative theory.

For this end, an interaction term of the Lagrangian density of a weakly interacting Dirac particle has been constructed [2]. The Lagrangian density obtained therein describes weak interactions between two spin-1/2 Dirac particles which are mediated by an appropriate weak field. The theory abides by the dimensions of the Fermi coupling constant $G_{F}$ and by its universality. It is proved in [2] that this interaction term is consistent with the vector $\boldsymbol{V}$ and the axial-vector $\boldsymbol{A}$, which are two attributes of weak interactions and with their associated parity nonconservation processes. The present work contains a further investigation of the system and proves new properties of the equations of weakly interacting particles and of their mediating fields.

Units where $\hbar=c=1$ are used. Greek indices run from 0 to 3 and Latin indices run from 1 to 3 . The diagonal metric is $(1,-1,-1,-1)$. Square brackets [ ] denote the dimension of the enclosed expression. In a system of units where $\hbar=c=1$ there is just one dimension, and the dimension of length, denoted by $[L]$, is used. In particular, energy and momentum take the dimension $\left[L^{-1}\right]$ and the dimension of a dipole is $[L]$. Throughout this work the calligraphic letters $\mathcal{F}, \mathcal{B}, \mathcal{E}$ and $\mathcal{A}$ denote weak interactions quantities that are analogous to the corresponding electromagnetic quantities where $F^{\mu \nu}$ denotes the electromagnetic field tensor, $\boldsymbol{B}, \boldsymbol{E}$ denote the magnetic and the electric fields, respectively and $A_{\mu}$ is the 4-potential of the fields. The second section shows how the weak fields equations of motion can be derived from a Lagrangian density. The third section examines solutions of the weak fields equations of motion. Some properties of the equations of motion of weakly interacting Dirac particles are derived in the fourth section. A discussion of the results is presented in the fifth section. It shows similarity and differences between the weak interaction theory which is discussed below and quantum electrodynamics (QED) of an electrically charged Dirac particle. The last section contains a summary of the results.

\section{The Weak Fields Equations of Motion}

The neutrino is regarded here as a chargeless massive Dirac particle that carries a weak interaction elementary axial dipole. The weak fields of this dipole are analogous to the electromagnetic fields of an elementary axial dipole. Hence, the standard form of the Lagrangian density of the system is (see [2])

$$
\mathcal{L}_{w}=\bar{\psi}\left(i \gamma^{\mu} \partial_{\mu}-m\right) \psi-\frac{1}{16 \pi} \mathcal{F}^{\mu v} \mathcal{F}_{\mu v}+d \bar{\psi} \sigma_{\mu v} \mathcal{F}^{\mu v} \psi .
$$


Here $\mathcal{F}^{\mu v}$ is a $4 \times 4$ antisymmetric tensor of the weak fields, $d$ denotes the strength of an elementary weak dipole, and $\sigma_{\mu v}$ is defined in terms of the Dirac $\gamma$ matrices (see [3] p. 21)

$$
\sigma_{\mu v} \equiv \frac{i}{2}\left(\gamma_{\mu} \gamma_{v}-\gamma_{v} \gamma_{\mu}\right) .
$$

The first term of (1) describes a free Dirac particle (see [4], p. 54). The second term is that of the free weak fields, whose structure is analogous to that of free electromagnetic fields (see [4], p. 70). The third term represents the dipole-fields weak interaction (see [2], Equation (11)).

An application of the variational principle to the mediating fields $\mathcal{F}^{\mu v}$ of (1) yields their equations of motion. The analysis of the electromagnetic fields (see [1]) indicates how to accomplish this task. Like the case of the electromagnetic fields, the weak fields $\mathcal{F}_{\mu \nu}$ are the 4-curl of a 4-potential $\mathcal{A}_{\mu}$ (see [1], p. 65)

$$
\mathcal{F}_{\mu v} \equiv \mathcal{A}_{v, \mu}-\mathcal{A}_{\mu, v} \text {. }
$$

Following the analysis of [1] (see pp. 70-71), one obtains the homogeneous equations

$$
\mathcal{F}_{, v}^{* \mu v}=0 .
$$

Here $\mathcal{F}^{* \mu v}$ is the dual tensor of $\mathcal{F}^{\mu v}$

$$
\mathcal{F}_{\mu v}^{*} \equiv \epsilon_{\mu v \alpha \beta} \mathcal{F}^{\alpha \beta}
$$

and $\epsilon_{\mu v \alpha \beta}$ is the completely antisymmetric unit tensor of the fourth rank. The equations (4) are derived from the mathematical structure of (3). They correspond to the homogeneous pair of Maxwell equations and are independent of the Lagrangian density (1) (see [1], pp. 70-71).

Another pair of equations is derived from the Lagrangian density (1). The general form of the Euler-Lagrange equations is (see [4], p. 15)

$$
\frac{\partial \mathcal{L}}{\partial \varphi_{r}}-\frac{\partial}{\partial x^{\mu}} \frac{\partial \mathcal{L}}{\partial\left(\partial \varphi_{r} / \partial x^{\mu}\right)}=0
$$

Here $\varphi_{r}, r=1, \cdots, N$ denotes the $r$ th independent fields of the system and the Equation (6) are obtained "by independently varying each field, $\delta \varphi_{r}(x)$ " (see [4], p. 15). It is explained in the following lines how an application of (6) to the weak fields of (1) yields the following equations

$$
\mathcal{F}_{, v}^{\mu v}=0 \text {. }
$$

This is the second pair of the equations of motion of the weak fields.

These equations are obtained from a procedure that is analogous to the derivation of the inhomogeneous pair of Maxwell equations (see [1], pp. 78-79). However, the interaction term of the weak Lagrangian density (the last term of (1)) differs from the standard form of the interaction term of the electromagnetic fields (see [4], p. 84)

$$
\mathcal{L}_{\text {int }}^{\mathrm{QED}}=-e \bar{\psi} \gamma^{\mu} A_{\mu} \psi
$$

It follows that the electromagnetic interaction term depends on the compo- 
nents of the 4-potential $A_{\mu}$ which are regarded as the generalized coordinates, whereas the weak interaction term depends on the derivatives of $A_{\mu}$, namely the weak fields $\mathcal{F}^{\mu v}$ of (3). In the electromagnetic case, the variation of the fields $A_{\mu}$ yields the inhomogeneous term of Maxwell equations (see [1], p. 79)

$$
F_{, v}^{\mu \nu}=-4 \pi e \bar{\psi} \gamma^{\mu} \psi,
$$

where $e \bar{\psi} \gamma^{\mu} \psi$ is the quantum expression for the 4-current $j^{\mu}$ of a Dirac particle (see [3], pp. 23, 24).

By contrast, the weak interaction term of (1) is independent of the function $A_{\mu}$. Hence, the Euler-Lagrange Equations (6) and the linear dependence of the weak interaction term on the fields' derivatives $\mathcal{F}^{\mu \nu}$ of (3) yield a null value. Hence, the second pair of the equations of motion of the weak fields (7) is homogeneous

$$
\mathcal{F}_{, v}^{\mu v}=0 .
$$

This analysis proves that the Maxwellian-like equations of motion of the weak fields, which are used in [2], can be consistently derived from an appropriate Lagrangian density.

\section{Solutions of the Weak Fields Equations of Motion}

It is proved in Section 2 that the equations of motion of the weak fields take the form of the four homogeneous Maxwell equations. Therefore, the vast literature on mathematical properties of electromagnetic fields is useful for finding solutions to the weak fields.

The simplest case is that of the weak fields which are calculated in the rest frame of a Dirac particle, say a neutrino. Here we have a motionless elementary pointlike axial dipole located at the origin of coordinates. As is well known, at far enough regions this field takes the form of the magnetic field of a localized system of stationary currents (see [1], pp. 111-113 or [5], pp. 180-182). Let d denote moment of the system's magnetic dipole. At a far enough point $\boldsymbol{x}$, the required 3-dimensional vector potential is

$$
A(x)=\frac{d \times x}{|x|^{3}}
$$

and its magnetic field is

$$
B(x)=\frac{3 x(x \cdot d)-|x|^{2} d}{|x|^{5}} .
$$

Having this information, one can apply a mathematical approach and examine a sequence of localized systems of stationary electric currents that have the same magnetic moment $\boldsymbol{d}$ and their spatial size tends to zero. Thus, concerning the fields of a motionless pointlike axial dipole of a Dirac particle, one finds that the validity of the 4-potential (11) and of its fields (12) holds for any given point near the origin. This argument proves that (11) and (12) hold for the entire 
3-dimensional space outside the origin.

The structure of the weak 4-potential (11) differs from that of the electromagnetic case of a charge $e$. Thus, in the electromagnetic case, the 4-potential of an electric charge $e$ is given by the Lienard-Wiechert formula which takes the following 4-vector form (see [1], p. 174)

$$
A_{\mu}=e \frac{v_{\mu}}{R^{\mu} v_{\mu}} .
$$

Here $R^{\mu}$ denotes the distance from the space-time point where the 4-potential is measured to the retarded location of the charge and $v_{\mu}$ is the retarded 4-velocity of the charge.

Let us examine the fields at a large distance from the location of the particles. In the electromagnetic case the 4-potential (13) decreases like $1 / R$ whereas in the weak case $(11)$ it decreases like $1 / R^{2}$. The corresponding fields are obtained from the 4-curl (3) of the 4-potential. Assume that a given function $f(t, \boldsymbol{r})$ decreases at a large distance like $1 / r^{n}$. Then, its derivative with respect to a spatial coordinate decreases like $1 / r^{n+1}$ whereas its derivative with respect to time decreases like $1 / r^{n}$. It follows that derivatives with respect to time are dominant at large distance. Thus, the 4-potential of the electromagnetic fields (13) proves that its time derivative decreases like $1 / r$ whereas the weak 4 potential (11) as well as its time-derivative decrease like $1 / r^{2}$. These properties are useful for finding the radiation components of the fields.

The dimension of the given quantities helps us find the structure of expressions that represent energy radiation. Energy has the dimension $\left[L^{-1}\right]$ and density has the dimension $\left[L^{-3}\right]$. Hence, energy density has the dimension $\left[L^{-4}\right]$. Now, the electromagnetic fields and the weak fields have the dimension $\left[L^{-2}\right]$. For this reason an expression for energy density must depend quadratically on the fields. (The energy-momentum tensor of electromagnetic fields represents energy-momentum density and current. And indeed, its form is a product of the fields (see [1], p. 87 or [5], p. 605)). Furthermore, radiation intensity obeys the inverse square law, which means that it decreases like $1 / r^{2}$. Therefore, electromagnetic fields which have a component that decreases like $1 / r$ may emit radiation, because the product of such fields decreases like $1 / r^{2}$. On the other hand, weak fields, whose most significant term decreases like $1 / r^{2}$ cannot emit radiation.

This result indicates that:

\section{a system of weak dipoles does not emit radiation.}

The foregoing discussion holds for a motionless weak dipole. Hence, in order to prove this claim one has to examine the general case of fields of an arbitrarily moving weak dipole. The fact that the weak fields satisfy the four homogeneous Maxwell equations is useful for this task. Thus, the following proof uses mathematical information which is found in textbooks that discuss Maxwellian electrodynamics. In particular, the validity of statements used below for the proof is substantiated merely by an appropriate reference to specific pages of 
textbooks.

Let us define the origin of coordinates at the instantaneous position of a given Dirac particle and use spherical polar coordinates where the $\mathrm{z}$-axis is parallel to the instantaneous direction of the particle's spin. The homogeneous Maxwell equations prove that each component of the 4-potentials satisfies the free wave equation (see [1], pp. 171-172, Equations (62.2) and (62.6))

$$
\Delta A_{\mu}-\frac{\partial^{2} A_{\mu}}{\partial t^{2}}=0 .
$$

In spherical polar coordinates one finds the following expression for the Laplacian

$$
\Delta \equiv \frac{1}{r^{2}} \frac{\partial}{\partial r}\left(r^{2} \frac{\partial}{\partial r}\right)-\frac{1}{r^{2}} \boldsymbol{I}^{2}
$$

where $\boldsymbol{I}^{2}$ is the square of the angular momentum operator (see [6], pp. 107108; [7], p. 15). The following analysis determines the required properties of solutions to the wave Equation (14).

Let us examine the solution at a region of space which is close to the position of the particle. As stated in [1] (see near the bottom of p. 172), at this region "the derivatives with respect to the coordinates increase more rapidly than its time derivative." Therefore, near the origin of coordinates the solution of the wave equation (14) is determined by the spatial derivatives. It follows that at this region the solution takes the form of the static dipole (11) and it varies like $r^{-2}$. The Laplacian operator (15) of the wave Equation (14) is homogeneous with respect to $r$ and its order is $r^{-2}$ whereas the time derivative operator of (14) is homogeneous with respect to $r$ and its order is $r^{0}$. It follows that the radial dependence of a solution to the wave Equation (14) may have terms that behave like $r^{-N}$, where $N \geq 2$.

As stated above, energy density depends quadratically on the fields. Therefore, the radial dependence of the weak field, which decreases like $r^{-N}$, where $N \geq 2$, means that at a large distance the weak fields' energy density decreases like $r^{-4}$, or even faster. This result proves that weak fields have no radiation term, because radiation obeys the inverse square law.

It is interesting to compare the radiationless weak axial dipole fields with the magnetic dipole radiation of electrodynamics. Indeed, in the case of weak field, the primary expression of the 4-potential (11) decreases like $1 / r^{2}$. Hence, as shown above, its fields at far enough regions cannot have a radiation component. On the other hand, the magnetic dipole radiation of electrodynamics is obtained from a power series expansion of the primary expression for the retard 4potential (see [1], pp. 203-205 or [5], pp. 397-398). The electromagnetic 4potential (13) decreases like $1 / r$. Therefore, the calculation shows that its power series expansion also contains terms that decrease like $1 / r$. In the general case, these terms determine the magnetic dipole contribution to electromagnetic radiation. 


\section{Equations of Motion of Weakly Interacting Particles}

The Lagrangian density (1) of the system also determines the equations of motion of a weakly interacting Dirac particle. For this end one has to vary only the fields that describe this particle (see e.g. [4], p. 84). The second term of the Lagrangian density (1) depends only on the fields that mediate weak interactions. Therefore, it is ignored in the calculation of the particle's equations of motion.

The interaction term of the Lagrangian density of a weakly interacting particle (1) is

$$
\mathcal{L}_{\text {int }}=d \bar{\psi} \sigma_{\mu v} \mathcal{F}^{\mu v} \psi .
$$

The operator $d \sigma_{\mu \nu} \mathcal{F}^{\mu v}$ of (16) is analogous to the operator of the electromagnetic interaction of the electron $-e \gamma^{\mu} A_{\mu}$. Indeed, each of them is a Lorentz scalar whose dimension is $\left[L^{-1}\right]$. In the electromagnetic case, quantum properties of the electron can be examined in circumstances that can be divided into two different categories-bound states and processes where the electron is free.

A bound state of the electron has a well defined energy and in the Schroedinger picture the time coordinate of its wave function appears only in the factor $\exp (-i E t)$, where $E$ denotes the energy of the given state (see [6], p. 28). Considering a specific energy state $\psi_{n}$, one finds that the electron's energy is an eigenvalue of the Hamiltonian and it is derived from the Dirac equation (see e.g. $[6]$, p. 28 or [3], p. 52)

$$
E_{n}=\int \psi_{n}^{\dagger} i \frac{\partial}{\partial t} \psi_{n} \mathrm{~d}^{3} r=\int \psi_{n}^{\dagger} H_{D} \psi_{n} \mathrm{~d}^{3} r
$$

where

$$
\psi^{\dagger} \gamma^{0} \equiv \bar{\psi}
$$

(see [3], p. 24) and $H_{D}$ denotes the Dirac Hamiltonian. Here $\psi_{n}^{\dagger}$ is the complex conjugate function of $\psi_{n}$ and both are the Hamiltonian's eigenfunctions which describe the same bound state of the electron.

In a scattering process the state $\psi_{f}$ of the outgoing electron differs from its incoming state $\psi_{i}$. The entire process is obtained from the time-integral of the intensity of the transition. It is described by the following matrix element (see [8], p. 186)

$$
M_{i f}=\int \psi_{f}^{\dagger} H_{D} \psi_{i} \mathrm{~d}^{3} r \mathrm{~d} t
$$

(This time integral is unnecessarily omitted from Equation (12) of [2]). Evidently, a free electron does not change its state. Therefore, only the interaction term of $H_{D}$ is used in actual calculations of (19).

Let us turn to the neutrino's weak interactions. Experiment does not show bound states of a neutrino. Therefore, only scattering processes are relevant to this comparison with electrodynamics. Like in the case of electron scattering (19), one must define the form of the Hamiltonian's interaction term which is derived from the Lagrangian term (16). The function $\psi^{\dagger}$ is used in the Hamiltonian and the additional $\gamma^{0}$ of (18) changes dramatically the tensorial 
structure of the weak interaction term of (16). Detailed calculations (see [2], Section 3) yield the following expression of the Hamiltonian's weak interactions term

$$
\psi^{\dagger} H_{i n t} \psi=d \psi^{\dagger} \gamma^{0} \sigma_{\mu \nu} \mathcal{F}^{\mu v} \psi=2 d \psi^{\dagger}\left(i \gamma_{i} \mathcal{E}^{i}-\gamma^{5} \gamma_{i} \mathcal{B}^{i}\right) \psi
$$

(The pure imaginary number $i$ should be distinguished from the running index i.) Here $\mathcal{E}^{i}, \mathcal{B}^{i}$ are the electric-like and magnetic-like 3-vectors of the corresponding entries of the antisymmetric tensor of the weak field (see [1], p. 65)

$$
\mathcal{F}^{\mu v}=\left(\begin{array}{cccc}
0 & -\mathcal{E}_{x} & -\mathcal{E}_{y} & -\mathcal{E}_{z} \\
\mathcal{E}_{x} & 0 & -\mathcal{B}_{z} & \mathcal{B}_{y} \\
\mathcal{E}_{y} & \mathcal{B}_{z} & 0 & -\mathcal{B}_{x} \\
\mathcal{E}_{z} & -\mathcal{B}_{y} & \mathcal{B}_{x} & 0
\end{array}\right) .
$$

Expression (20) which describes weak interactions comprises two terms. The $i \gamma_{i}$ of the first term are three Hermitian matrices which are entries of a 3 -vector and the three $\gamma^{5} \gamma_{i}$ of the second term are three Hermitian matrices which are entries of an axial 3-vector. They respectively correspond to the vector $\boldsymbol{V}$ and the axial vector $\boldsymbol{A}$ of weak interactions. In (20) the vector $i \gamma_{i}$ and the axial vector $\gamma^{5} \gamma_{i}$ are contracted with the appropriate weak fields $\mathcal{E}^{i}, \mathcal{B}^{i}$ of the other particle. Here the role of the fields of the other particle is analogous to the well-known example of the hydrogen atom, where the electron interacts with the $A_{0}$ static potential of the proton. Evidently, during the scattering process the weak fields $\mathcal{E}^{i}, \mathcal{B}^{i}$ as well as the entries of the spinors of the two interacting Dirac particles are functions of the space-time coordinates. In order to calculate a specific neutrino scattering process one must substitute the weak interaction term of (20) into the formula of the scattering process (19) and carry out the integrals over the space-time coordinates. These integrals yield appropriate coefficients of the 3-vector $i \gamma_{i}$ and of the axial 3-vector $\gamma^{5} \gamma_{i}$ which operate on the Dirac function $\psi$.

\section{Discussion}

The weak interaction theory which is discussed in this work is derived from an application of the variational principle to the Lagrangian density (1). The results show that the theoretical basis of weak interactions is similar to that of QED of an electrically charged Dirac particle. QED of such a particle relies on a well-known Lagrangian density $\mathcal{L}_{\mathrm{QED}}$ (see [4], p. 84). Let us write its standard form together with the weak interaction Lagrangian density $\mathcal{L}_{w}$ of (1), in order to facilitate a comparison between them.

$$
\begin{aligned}
& \mathcal{L}_{\mathrm{QED}}=\bar{\psi}\left(i \gamma^{\mu} \partial_{\mu}-m\right) \psi-\frac{1}{16 \pi} F^{\mu v} F_{\mu v}-e \bar{\psi} \gamma^{\mu} A_{\mu} \psi \\
& \mathcal{L}_{w}=\bar{\psi}\left(i \gamma^{\mu} \partial_{\mu}-m\right) \psi-\frac{1}{16 \pi} \mathcal{F}^{\mu v} \mathcal{F}_{\mu \nu}+d \bar{\psi} \sigma_{\mu \nu} \mathcal{F}^{\mu v} \psi .
\end{aligned}
$$


The QED Lagrangian density $\mathcal{L}_{\mathrm{QED}}$ of (22) comprises three terms: The first term, which contains the quantities enclosed in parentheses, describes a free Dirac particle. The second term describes free electromagnetic fields. The last term describes the interaction between an electrically charged Dirac particle and electromagnetic fields. Similarly, the Lagrangian density $\mathcal{L}_{w}$ of (1) is used in this work as a basis for the weak interaction theory. Like $\mathcal{L}_{\mathrm{QED}}$ of (22), it comprises three analogous terms: The first term is the same as its counterpart of (22) and it describes a free Dirac particle. The second term which describes free weak fields, takes the same mathematical form as that of the free electromagnetic fields of (22). The last term describes the weak interactions between a Dirac particle and the weak fields.

The form of the electromagnetic interaction term of a Dirac particle $-e \bar{\psi} \gamma^{\mu} A_{\mu} \psi$ abides by the following experimentally confirmed properties of Maxwellian electrodynamics: the dimensionless of the electric charge $\left[e^{2}\right]=\left[L^{0}\right]$ and its universality. Therefore, its operator $e \gamma^{\mu} A_{\mu}$ is a Lorentz scalar whose dimension is $\left[L^{-1}\right]$ and the dimension of $-e \bar{\psi} \gamma^{\mu} A_{\mu} \psi$ is $\left[L^{-4}\right]$. The other terms of (22) also have these properties. Indeed, having the same dimension and the same form of Lorentz transformation is a fundamental requirement that should be satisfied by any physical expression that contains several terms. Analogously, the weak interaction term of (1) abides by the following experimentally confirmed properties of weak interactions: the dimension of the Fermi constant $\left[G_{F}\right]=\left[L^{2}\right]$ and its universality. On this basis it is proved in [2] that weak interactions can be described as an interaction between axial dipoles of two Dirac particles. Here the dimension of each dipole is $[L]$ and the coupling constant that describes the interaction strength has the dimension $\left[L^{2}\right]$, which is the dimension of the Fermi constant $G_{F}$. For this reason, also the weak interaction operator of (1) $d \sigma_{\mu \nu} \mathcal{F}^{\mu v}$ is a Lorentz scalar whose dimension is $\left[L^{-1}\right]$. Indeed, the dimension of the dipole strength $d$ is $[L]$ and that of the weak fields $\mathcal{F}^{\mu v}$ is $\left[L^{-2}\right]$.

The analysis presented in [2] proves the following difference between electromagnetic fields and weak fields: as is well known, the electromagnetic fields satisfy two homogeneous Maxwell equations and two inhomogeneous Maxwell equations whereas in the case of weak fields the equations of motion take the form of the four homogeneous Maxwell equations.

The analysis of Section 3 yields the mathematical structure of the weak interactions fields. The following argument explains the similarity and the differences between electromagnetic fields and weak fields. Maxwellian electrodynamics contains the two inhomogeneous Equations (9). As explained in the Introduction section of [2], a field function of a given quantum field theory takes the form $\psi(x)$, where $x$ denotes the four space-time coordinates. This field function describes a pointlike particle.

Let us use these properties for an examination of a quantum system that comprises a finite number of electrically charged Dirac particles and for a comparison between it and a corresponding system of weakly interacting Dirac 
particles. For every Dirac particle define a small sphere whose center is at the particle's location and consider the entire three dimensional space $S^{\prime}$ outside these spheres. Evidently, the 4-current of the charges vanishes at $S^{\prime}$. Therefore, Maxwell equations at $S^{\prime}$ take the form of four homogeneous equations. It follows that at $S^{\prime}$ Maxwell equations takes the same form as the equation of motion of the mediating fields of weak interactions which is discussed in this work. This result means that at $S^{\prime}$ the electromagnetic 4-potentials and the weak 4-potentials solve the same wave equation (14). Specific solutions of these equations depend on the boundary values of the fields. In both cases the boundary values vanish at infinity. It follows that the difference between electromagnetic fields and weak fields stem from the different boundary values on the small spheres that enclose the Dirac particles of the given system. In the electromagnetic case the boundary values are independent of the angular coordinates and the solution fits the Coulomb formula where the potential decreases like $1 / R$. On the other hand, in the weak interaction case the boundary values of the 4-potentials fit the cross product $\boldsymbol{d} \times \boldsymbol{X}$ of (11) which depends on the angular coordinates. It means that the solution fits the axial dipole formula (11) which decreases like $1 / R^{2}$.

Maxwellian electrodynamics and the weak interaction theory discussed here are related to two different attributes of a Dirac particle. The electric charge is related to the particle's density, which is the 0-component of the 4-current $e \bar{\psi} \gamma^{\mu} \psi$. The weak interaction theory discussed herein is related to the spin of a Dirac particle and its interaction term $d \bar{\psi} \sigma_{\mu \nu} \mathcal{F}^{\mu v} \psi$ yields fields of an axial dipole. It turns out that the associated Hamiltonian which is derived from this weak interactions Lagrangian density comprises two terms, a vector $\boldsymbol{V}$ and an axial vector $\boldsymbol{A}$. This result fits weak interactions data where parity is not conserved.

\section{Concluding Remarks}

This work shows that the dynamics of weak interactions can be described as a dipole-dipole interaction between two Dirac particles. This theory relies on the experimentally confirmed finite mass of the neutrino [9] [10]. The equations of motion of its mediating fields take the form of four homogeneous Maxwell equations.

The basis of this weak interaction theory is the Lagrangian density (1) which is similar to the QED Lagrangian density (22) (see Section 5). This similarity indicates that the weak interaction theory described herein follows a successful route. The structure of this theory is consistent with the form of the Fermi weak interaction coupling constant $G_{F}$. The results prove parity violation in weak interactions processes. It is well known that this outcome is consistent with experimental data (see [8] pp. 214-215 and 220-222). This is probably the first time that parity violation of weak interactions processes is derived from general considerations which rely on the variational principle. Another result is derived in Section 3, which proves that the weak interactions mediating fields have no radiation term. 


\section{References}

[1] Landau, L.D. and Lifshitz, E.M. (2005) The Classical Theory of Fields. Elsevier, Amsterdam.

[2] Comay, E. (2016) A Theory of Weak Interaction Dynamics. Open Access Library Journal, 3, 1-10. https://doi.org/10.4236/oalib.1103264

[3] Bjorken, J.D. and Drell, S.D. (1964) Relativistic Quantum Mechanics. McGraw-Hill, New York.

[4] Bjorken, J.D. and Drell, S.D. (1965) Relativistic Quantum Fields. McGraw-Hill, New York.

[5] Jackson, J.D. (1975) Classical Electrodynamics. John Wiley, New York.

[6] Landau, L.D. and Lifshitz, E.M. (1959) Quantum Mechanics. Pergamon, London.

[7] de-Shalit, A. and Talmi, I. (1963) Nuclear Shell Theory. Academic Press, New York.

[8] Perkins, D.H. (1987) Introduction to High Energy Physics. Addison-Wesley, Menlo Park.

[9] Patrignani, C., et al. (Particle Data Group) (2016) Review of Particle Physics. Chinese Physics C, 40, 100001. https://doi.org/10.1088/1674-1137/40/10/100001

[10] Formaggio, J.A. and Zeller, G.P. (2012) From eV to EeV: Neutrino Cross Sections across Energy Scales. Reviews of Modern Physics, 84, 1307.

https://doi.org/10.1103/RevModPhys.84.1307

Submit or recommend next manuscript to OALib Journal and we will provide best service for you:

- Publication frequency: Monthly

- 9 subject areas of science, technology and medicine

- Fair and rigorous peer-review system

- Fast publication process

- Article promotion in various social networking sites (LinkedIn, Facebook, Twitter, etc.)

- Maximum dissemination of your research work

Submit Your Paper Online: Click Here to Submit

Or Contact service@oalib.com 